\title{
A New Variable Step-Size NLMS Algorithm and its Performance Evaluation in Echo Cancelling Applications
}

\author{
F. M. Casco-Sánchez ${ }^{* 1}$, R. C. Medina-Ramírez ${ }^{2}$, M. López-Guerrero ${ }^{3}$ \\ ${ }^{1,2,3}$ Departamento de Ingeniería Eléctrica \\ Universidad Autónoma Metropolitana \\ Av. San Rafael Atlixco 186, Col. Vicentina, 09340 \\ Iztapalapa, Mexico \\ *alfa@xanum.uam.mx
}

\begin{abstract}
In this work we introduce a variable step-size normalized LMS algorithm for adaptive echo cancellation in a FIR structure. In the proposed scheme, the step-size adjustment is controlled by using the square of the cross-correlation between the squared output error and the adaptive filter output. The proposed algorithm (that we call VSSSC after variable step size based on the squared cross-correlation) was evaluated using white noise and speech signals. Simulation results show that our proposal achieves better performance than similar algorithms in single and double talk. The proposed algorithm can be used in a number of applications such as in echo reduction for long-haul voice communications.
\end{abstract}

Keywords: LMS, step size, echo cancellation, system identification.

\section{RESUMEN}

En este trabajo presentamos un algoritmo LMS normalizado de paso variable para la cancelación adaptable de eco en una estructura FIR. En el esquema propuesto, el ajuste del tamaño del paso se controla usando el cuadrado de la correlación cruzada entre el cuadrado del error de salida y la salida del filtro adaptable. El algoritmo propuesto, que llamamos VSSSC (de las palabras en inglés variable step size based on the squared cross-correlation) se evaluó usando ruido blanco y señal de voz. Los resultados de simulación muestran que nuestra propuesta logra un mejor desempeño en comparación con algoritmos similares tanto en el caso de un hablante como cuando existe traslape en la conversación de dos hablantes. El algoritmo propuesto se puede utilizar en varias aplicaciones tales como la reducción de eco en comunicaciones de voz de larga distancia.

\section{Introduction}

Adaptive filters have been used in a large number of applications such as system identification, channel equalization, noise reduction and echo cancelling. Among such applications, echo cancelling is of paramount importance in the transmission of voice conversations across long distances.

In electrical terms, echo can be defined as a delayed and distorted version of the transmitted signal that is reflected back from the receiver to the source. Such phenomenon is more noticeable in long-haul communications where it makes voice conversations less intelligible. In order to deal with this problem, communication links make use of echo cancellers which are placed at the destination end point. The echo canceller estimates the magnitude of the echo component in the signal to be propagated back to the originator end point and subtracts it. Such procedure generates a residual echo that is used by an adaptive filter in order to adjust the echo canceller and decrease the estimation error.

Adaptive filters must have fast convergence rates and produce low estimation errors in order to be successfully used in echo cancellers and in many other applications. A landmark in the development of adaptive filters was the introduction of the least mean square (LMS) algorithm by Widrow and Hoff in 1960 [1]. This algorithm has found diverse applications due to its simplicity and robustness that has also made it the preferred benchmark in performance evaluation studies [2]. Furthermore, there is a wide availability of related studies 
including analytical models for prediction of its performance under different input conditions. However, the LMS algorithm updates the filter coefficients through a fixed-size adaptation step which may lead to long convergence times. A great deal of current research effort is aimed at increasing convergence rates and reducing the steady-state estimation error. For instance, Raymond et al. in [3] proposed a variable step size (VSS) LMS algorithm in which the step size adjustment is controlled by the square of the output error, providing less misadjustment and faster tracking than conventional LMS. Mayyas et al. in [4] proposed a variable step-size LMS algorithm that provides fast convergence at early stages of the adaptation. As a final example, we can mention the algorithm by Wee-Peng et al. [5] who proposed a new class of variable step LMS algorithms with reduced complexity but good performance. Additional work on this area has more recently been reported in [6] and [7].

In this paper, we introduce and evaluate a variablestep-size algorithm in which the step size adjustment is controlled by the square of the crosscorrelation between the squared output error and the adaptive filter output. We call this algorithm VSSSC after variable step size based on the squared cross-correlation. The remaining of this paper is organized as follows. In Section 2, we provide an overview of related work and we also define the metrics of performance to be used in this work. In Section 3, we describe the proposed VSSSC algorithm. In Section 4, we present simulation results that compare the performance of the proposal against the VSS and the conventional normalized LMS. Finally, in Section 5, we provide some conclusions.

\section{Background concepts and related work}

Consider a system identification configuration as depicted in Figure 1. In such a system the $(N \times 1)$ vector $\widehat{\boldsymbol{H}}(n)$ contains the coefficients of the adaptive filter. According to the NLMS algorithm, at the $(n+1)$ st iteration the filter coefficients are updated as follows:

$$
\widehat{\boldsymbol{H}}(n+1)=\widehat{\boldsymbol{H}}(n)+\beta_{N L M S}(n) e(n) \boldsymbol{X}(n)
$$

with

$$
\beta_{N L M S}(n)=\frac{\alpha}{\|\boldsymbol{X}(n)\|^{2}}
$$

and

$$
e(n)=d(n)-\boldsymbol{X}^{T}(n) \widehat{\boldsymbol{H}}(n)
$$

where $e(n)$ is the output error, $d(n)$ is the desired signal, $0 \leq \alpha \leq 1$ is a convergence factor that controls both stability and convergence rate, the $(N \times 1)$ vector $\boldsymbol{X}(n)$ contains the last $N$ samples of the input and $\|\boldsymbol{X}(n)\|^{2}$ is its norm.

Several variable step-size LMS algorithms have been proposed to achieve better performance figures than conventional NLMS algorithms. They provide smaller mean square error without restricting the tracking ability or reducing convergence rates. In this context, one algorithm worth mentioning is the one introduced by Raymond et al. in [3]. They proposed a variable step-size LMS algorithm (VSS) in which a convergence factor is proportional to the power of the output error. In this algorithm the filter coefficients are updated as follows (see Figure 2):

$$
\widehat{\boldsymbol{H}}(n+1)=\widehat{\boldsymbol{H}}(n)+\beta_{V S S}(n) e(n) \boldsymbol{X}(n)
$$

where parameter $\beta_{V S S}(n)$ is the step size and its value is updated as

$$
\beta_{V S S}(n+1)=\left\{\begin{array}{cc}
\alpha_{\max } & \text { if } \alpha(n+1)>\alpha_{\text {max }} \\
\alpha_{\min } & \text { if } \alpha(n+1)<\alpha_{\text {min }} \\
\alpha(n+1) & \text { otherwise }
\end{array}\right.
$$

where

$$
\alpha(n+1)=\lambda \alpha(n)+\gamma e^{2}(n)
$$

for $0 \leq \lambda \leq 1$ and $\gamma>0$. Raymond et al. [3] experimentally found that $\lambda=0.997$ and $\gamma=$ 0.00048 provide adequate performance. In (5), parameter $\alpha_{\max }$ is set to ensure that the algorithm remains stable and $\alpha_{\min }$ is fixed to avoid a too small convergence factor after convergence. 


\section{A New Variable Step-Size NLMS Algorithm and its Performance Evaluation in Echo Cancelling Applications, F. M. Casco-Sánchez et al. / 302-313}

A commonly used metric of performance to evaluate the performance of adaptive filters in echo cancelling applications is the echo return loss enhancement (ERLE). This metric given by [8].

$E R L E=\frac{S}{N}+10 \log _{10}\left(\frac{2}{\alpha}-1\right)$

where

$\frac{S}{N}=10 \log _{10}\left\{\frac{E\left[y^{2}(n)\right]}{E\left[r^{2}(n)\right]}\right\}$

is the signal-to-noise ratio between the signal $y(n)$ and noise $r(n)$. The noise is assumed to be uncorrelated with the input signal $x(n)$ [2].
Another commonly used metric of performance is convergence time.

This concept provides a measure related to how fast an algorithm is able to reach its steady-state value. We define the convergence time as the number of iterations that is needed for the ERLE metric to remain above $(1-\epsilon)$ times its steady-state value, for some given $\epsilon>0$.

A performance comparison between our proposal, described in the following section, and the algorithms described above will be carried out in Section 4, in terms of both ERLE and convergence time.

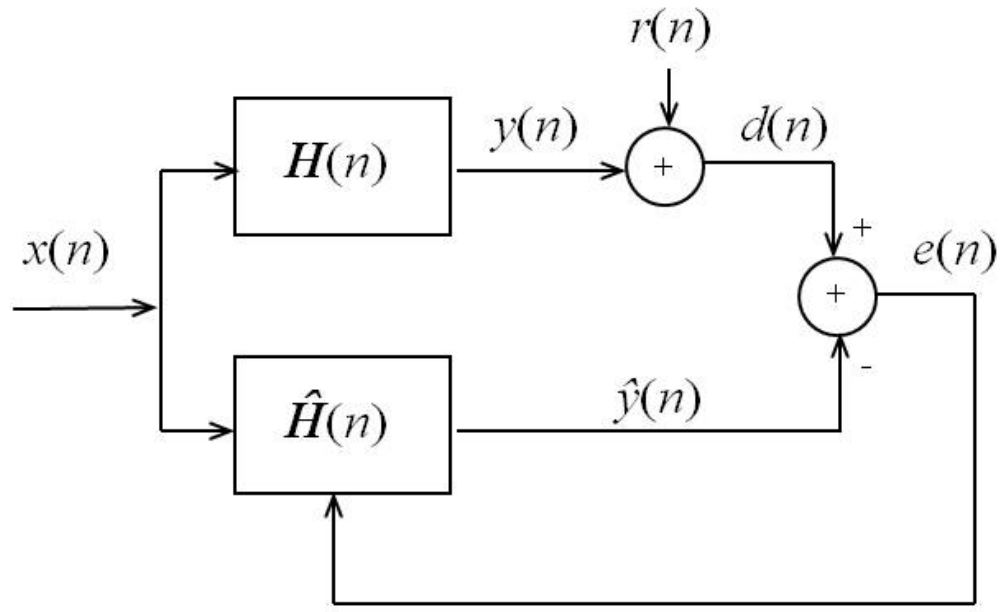

Figure 1. Normalized LMS algorithm in a system identification configuration. 


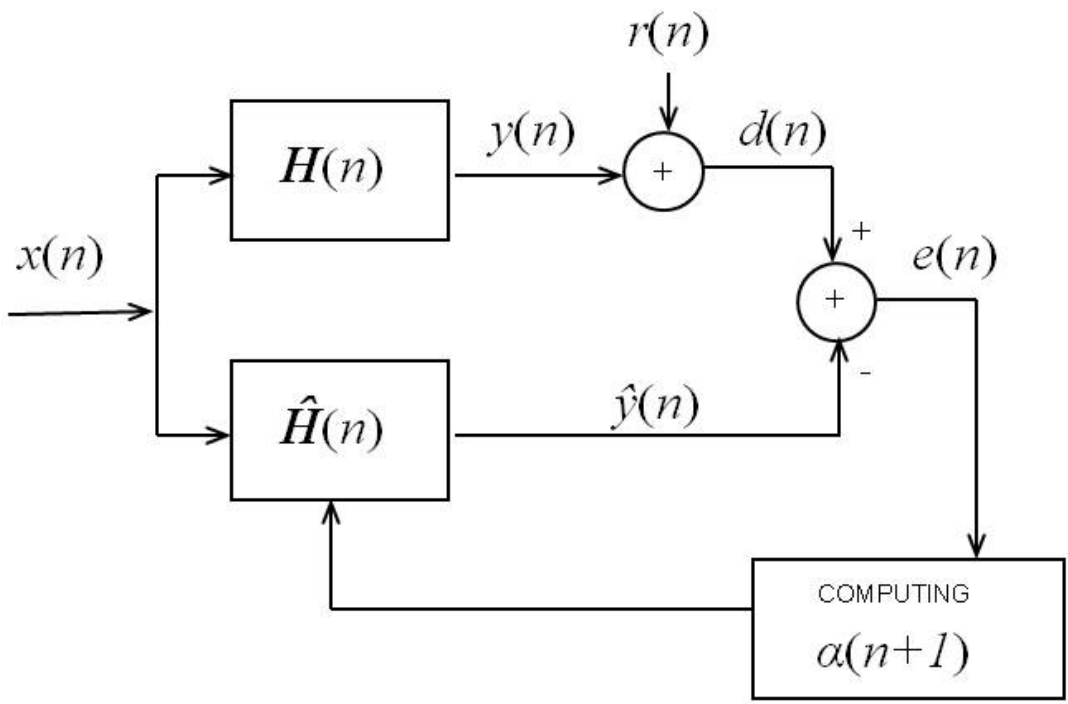

Figure 2. The VSS LMS algorithm in a system identification configuration.

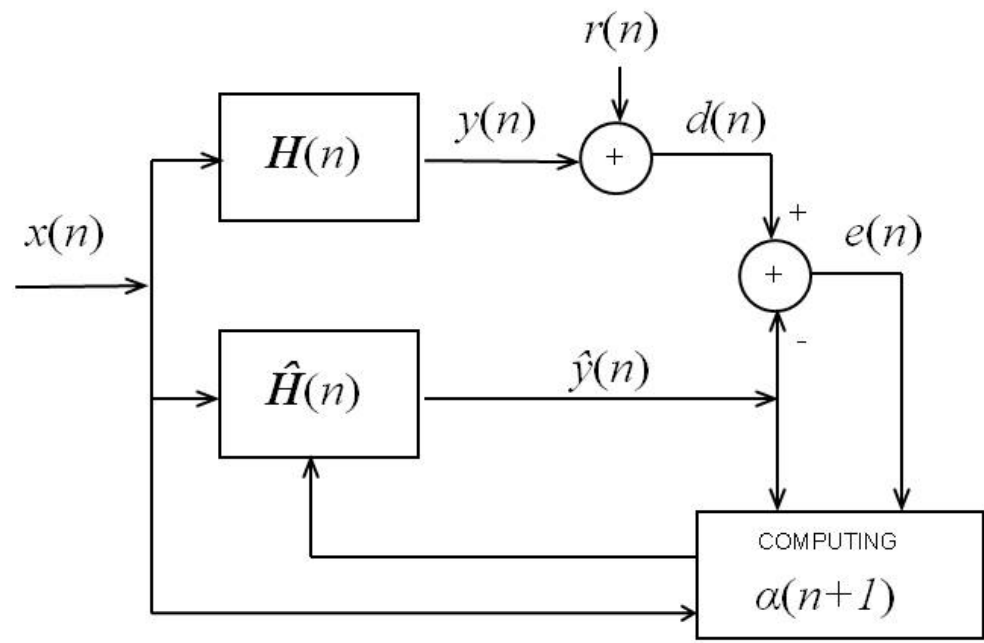

Figure 3. The VSSSC algorithm in a system identification configuration. 


\section{VSSSC: a variable-step-size NLMS algorithm}

Let us consider the system identification configuration depicted in Figure 3. The derivation of the proposed algorithm starts with the recursive equation

$$
\widehat{\boldsymbol{H}}(n+1)=\widehat{\boldsymbol{H}}(n)+\beta_{V S S S C}(n) e(n) \boldsymbol{X}(n)
$$

with

$e(n)=d(n)-\boldsymbol{X}^{T}(n) \widehat{\boldsymbol{H}}(n)$

where $\widehat{\boldsymbol{H}}(n), \quad \boldsymbol{X}(n)$ and $d(n)$ have the same meaning as in the NLMS algorithm previously explained. In order to update the step size, it is proposed to take into consideration the squared cross-correlation, at lag zero, between the squared output error and the filter output. This quantity is defined as follows:

$$
\begin{aligned}
R_{e^{2}, \hat{y}}^{2}(n) & =\left\{\left.E\left[e^{2}(n) \hat{y}(n+m)\right]\right|_{m=0}\right\}^{2} \\
& =\left\{E\left[e^{2}(n) \hat{y}(n)\right]\right\}^{2}
\end{aligned}
$$

and denote by $\hat{R}_{e^{2}, \hat{y}}^{2}(n)$ its estimated value by means of a time average. The step size is then updated as follows:

$\beta_{V S S S C}(n)=\frac{\alpha(n)}{\|X(n)\|^{2}}$

where we propose to update $\alpha(n)$ by using

$$
\alpha(n+1)=\left\{\begin{array}{cc}
\alpha_{\max } & \text { if } \alpha^{\prime}(n+1)>\alpha_{\max } \\
\alpha_{\min } & \text { if } \alpha^{\prime}(n+1)<\alpha_{\min } \\
\alpha^{\prime}(n+1) & \text { otherwise }
\end{array}\right.
$$

where

$\alpha^{\prime}(n+1)=\frac{\hat{R}_{e^{2}, \hat{y}}^{2}(n+1)}{\hat{x}^{2}(n+1)}$ with

$$
\hat{R}_{e^{2}, \hat{y}}^{2}(n+1)=\lambda \hat{R}_{e^{2}, \hat{y}}^{2}(n)+\gamma\left[e^{2}(n) \hat{y}(n)\right]^{2}
$$

and

$\hat{x}^{2}(n+1)=\lambda \hat{x}^{2}(n)+\gamma\left[x^{2}(n)\right]$

Parameter $\lambda$ satisfies

$$
\lambda \leq 1-\frac{1}{N}
$$

and $1 / \gamma$ is approximately equal to the number of samples $N$, used for estimating the averages, i.e.,

$$
\gamma=\frac{1}{N}
$$

Parameters $\lambda$ and $\gamma$ allow us to control the weight of past samples against the present ones as it has been done in a number of algorithms. In (13), parameter $\alpha_{\max }$ is set to one to ensure fast convergence and $\alpha_{\min }$ is fixed to avoid a too small convergence factor $\alpha$ (after convergence is achieved).

The rationale behind this algorithm can be explained as follows. From Figure 3, it can be seen that

$$
e(n)=y(n)-\hat{y}(n)+r(n)
$$

Recall that $\hat{y}(n)$ is the estimate for $y(n)$ and $r(n)$ is additive noise. From (11) and (19) we have

$$
R_{e^{2}, \hat{y}}^{2}(n)=\left(E\left\{[y(n)-\hat{y}(n)+r(n)]^{2} \hat{y}(n)\right\}\right)^{2}
$$

Note that at the beginning the squared correlation between $e^{2}(n)$ and $\hat{y}(n)$ is large because $[y(n)-\hat{y}(n)]$ is large too. Therefore, the step size will also be large (see (14)). On the other hand, when the algorithm converges, the correlation $\hat{R}_{e^{2}, \hat{y}}^{2}(n)$ decreases since $y(n) \cong \hat{y}(n)$ and (20) approximately becomes 


\begin{tabular}{|lccccc|}
\hline & $\alpha_{\max }$ & $\alpha_{\min }$ & $\lambda$ & $\gamma$ & $M$ \\
\hline NLMS & 1 & - & 0.997 & $4.8 \times 10^{-4}$ & 128 \\
VSS & 1 & 0.02 & 0.997 & $4.8 \times 10^{-4}$ & 128 \\
VSSSC & 1 & 0.02 & 0.997 & $4.8 \times 10^{-4}$ & 128 \\
\hline
\end{tabular}

Table 1. Simulation parameters.

\begin{tabular}{|l|c|}
\hline Algorithm & $\begin{array}{l}\text { Convergence time } \\
(\text { Iterations } \times 300)\end{array}$ \\
\hline VSSSC & 27 \\
VSS & 56 \\
NLMS & 4 \\
\hline
\end{tabular}

Table 2. Convergence-time comparison for nIms, VSS and VSSSC.

White noise as the input signal.

$$
\begin{aligned}
R_{e^{2}, \hat{y}}^{2}(n)=( & \left.E\left\{[y(n)-\hat{y}(n)+r(n)]^{2} \hat{y}(n)\right\}\right)^{2} \\
& \cong\left\{E\left[r^{2}(n) \hat{y}(n)\right]\right\}^{2}
\end{aligned}
$$

In such conditions, the step size is small since $r^{2}(n)$ and $\hat{y}(n)$ are almost uncorrelated.

\section{Performance evaluation}

In this section, we describe the experiments and the corresponding results that were obtained from the performance evaluation that was carried out with the NLMS, VSS and the proposed VSSSC.

In our experiments, the system to be identified had an impulse response $h(n)$ given by

$h(n)=\left\{\begin{array}{cc}1 & n=0 \\ S_{a}(M \pi / c) & n \neq 0\end{array}\right.$

where $M$ is the number of taps in the transversal filter and parameter $c$ is the cutoff frequency of the low pass filter in the frequency domain. Table 1 shows the parameters used in the simulations.
The ERLE metric given by (7) was estimated as

$\operatorname{ERLE}(n)=10 \log _{10}(\operatorname{SNR}(n))$

where

$\operatorname{SNR}(n+1)=\frac{d_{2}(n+1)}{e_{2}(n+1)}$

with

$d_{2}(n+1)=\lambda d_{2}(n)+\gamma d^{2}(n)$

and

$e_{2}(n+1)=\lambda e_{2}(n)+\gamma e^{2}(n)$

where we used $\lambda=0.997$ and $\gamma=0.00048$.

In addition to the ERLE metric we also took measurements of the convergence time for the three different algorithms under test. In our experiments, we used $\epsilon=0.1$ so that the convergence time corresponded to the number of iterations needed for the ERLE metric to be within $10 \%$ of its steady-state value. 
In what follows, we present the experiments that we carried out and their results. These tests are divided into two sets depending on the use of either white noise or voice signal as the input signal. They are described below.

\subsection{First set of results: experiments with white} noise

In the first set of experiments, both the input signal and the additive noise were white noise and we used an SNR of $35[\mathrm{~dB}]$. We report our results regarding convergence performance and steadystate error of the three algorithms, NLMS, VSS and VSSSC.

In these experiments, whose results are shown in Fig. 4, the proposed VSSSC algorithm began with a large $\alpha$ as did the conventional NLMS and the VSS. However, after several iterations the proposed algorithm provided better steady-state ERLE than both VSS and NLMS. The NLMS converged to the SNR used in the simulations (i.e., $35[\mathrm{~dB}])$.

The convergence times that were obtained for the three algorithms in these experiments are shown in Table 2. It is shown that the shortest convergence time was achieved by the NLMS algorithm whereas the longest one was exhibited by VSS. The convergence time of VSSSC was located at some point in between.
From this set of experiments, it can be concluded that there exists a trade-off between ERLE and convergence time. In comparison to VSS and NLMS, the VSSSC algorithm achieves the best performance in terms of short and long term ERLE. On the other hand, its fine step adjustment increases convergence time when compared to the fixed step approach (i.e., NLMS) but its performance is significantly better than the other variable step algorithm (i.e., VSS).

In Figure 5, we illustrate the evolution for one point of the adaptive impulse response $h_{a p}[50]$, using the proposed VSSSC algorithm. For comparison purposes, the corresponding point of the unknown system impulse response $h[50]$ is also shown. As shown in the Figure, at the beginning the difference between them was large but after a few iterations $h_{a p}$ [50] closely approximated $h[50]$.

In order to show the adaptability of the proposed algorithm, we also ran tests in which the impulse response of the system to be identified was changed at some point during the simulation. Figure 6 shows the convergence performance of NLMS, VSS and VSSSC algorithms in two periods (labeled as I and II in the Figure) with different impulse responses. In period $\mathrm{I}$, it is clear that the VSSSC algorithm achieved better figures of ERLE than the VSS algorithm, whereas the NLMS algorithm converged to the SNR ratio. After some iterations, the system's response changed and

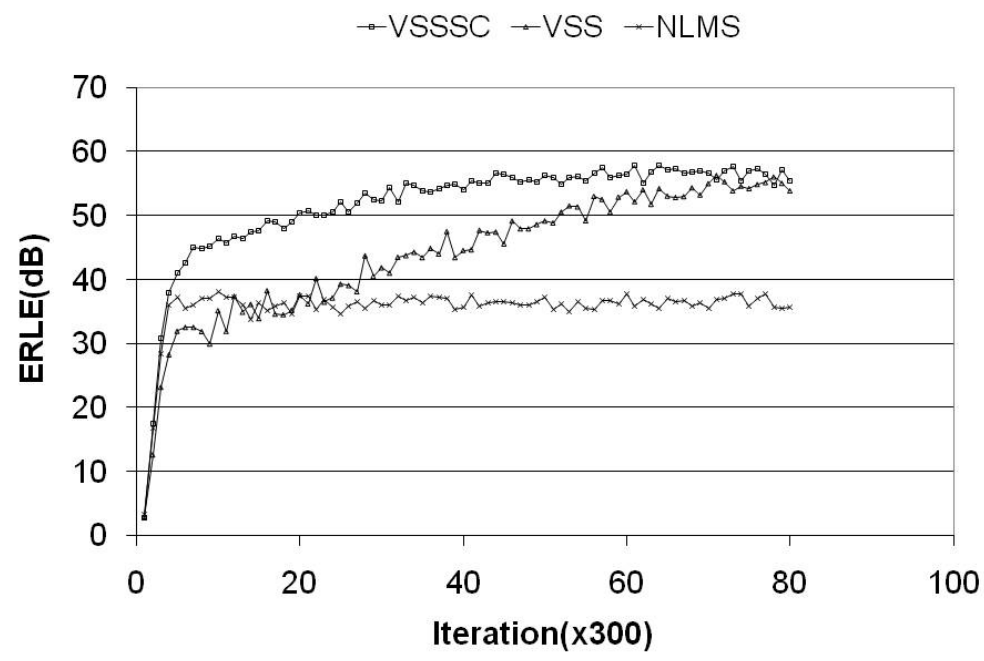

Figure 4. Convergence test of conventional NLMS, VSS, and VSSSC algorithms. 
period II began. When this change occurred, the VSSSC algorithm was using a small $\alpha$, but this value increased when the squared correlation between $\hat{y}(n)$ and $e^{2}(n)$ suddenly increased. Figure 6 shows that in period II the VSSSC algorithm again achieved better performance than the VSS algorithm. At the end of both periods the VSSSC achieved significantly better performance in terms of ERLE than both VSS and NLMS. Measurements of convergence time were not collected for this set of experiments since the nature of the tests does not allow the adaptive filters to reach steady state.

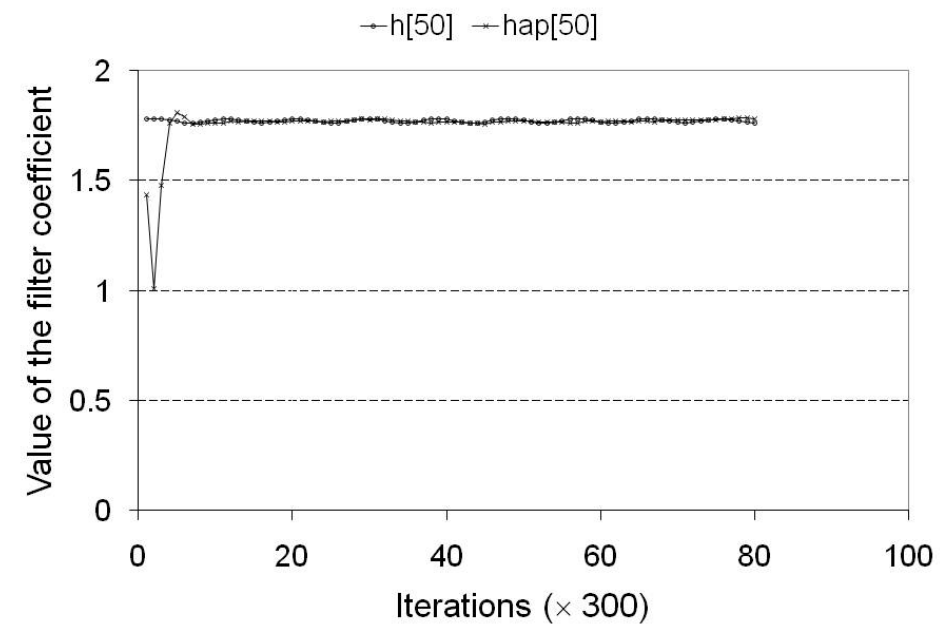

Figure 5. Evolution of point of the adaptive impulse response using the proposed VSSSC algorithm.

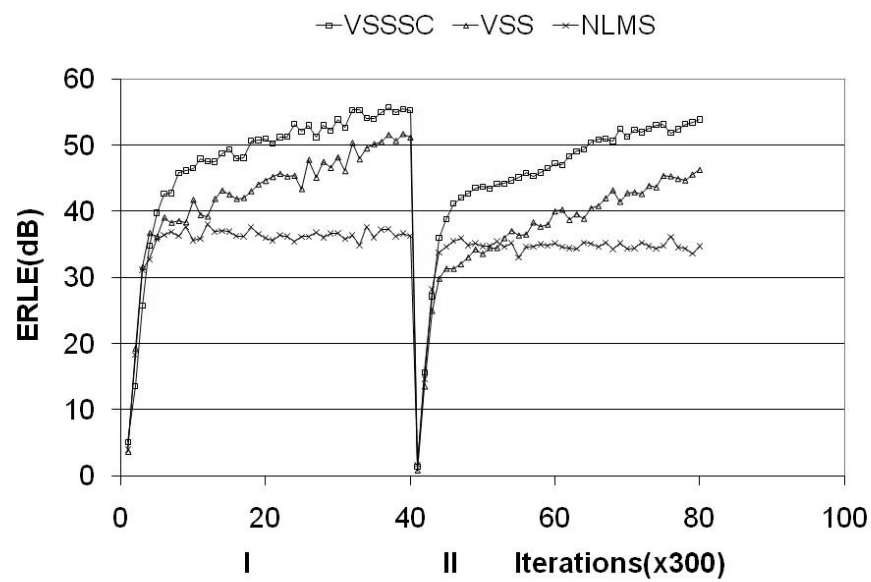

Figure 6. Convergence tests with conventional NLMS, VSS and VSSSC algorithms with a change in the impulse response. 


\begin{tabular}{|lc|}
\hline Algorithm & $\begin{array}{c}\text { Convergence time } \\
(\text { Iterations } \times 300)\end{array}$ \\
\hline VSSSC & 35 \\
VSS & 57 \\
NLMS & 7 \\
\hline
\end{tabular}

Table 3. Convergence-time comparison for NLMS, VSS and VSSSC.

Voice as the input signal signal.

4.2 Second set of results: experiments with a voice signal

In the second set of experiments, the received signal was a voice signal and the additive noise was white noise. We also used an SNR of 35 [dB]. Figure 7 shows the convergence performance of NLMS, VSS and VSSSC algorithms. After some iterations the proposed VSSSC algorithm achieved significantly better figures of ERLE than both VSS and NLMS. As in the previous set of results, the NLMS algorithm converged to the SNR ratio (i.e., 35 [dB]). Table 3 shows the convergence times that were measured for this set of tests. When compared with the results shown in Table 2, it is observed that there was a slight increase in convergence times when the input signal was changed from white noise to a real voice signal. However, the relative performance among the three algorithms remained practically the same.
Figure 8 illustrates the adaptability of NLMS, VSS and VSSSC algorithms when there is a change in the impulse response of the system to be identified. In the first period the performance of VSS is lower than the one shown by VSSSC, albeit similar in trend. However, after the system's response was changed, the VSS algorithm was unable to quickly react thus exhibiting a slow convergence rate. In both periods the NLMS algorithm converged to the level of SNR used in the experiments.

We close this section by noting that in both sets of experiments (i.e., white noise and realistic voice signals, as the input signal) we observed that the proposed VSSSC clearly outperforms the other two algorithms in terms of ERLE. As for convergence, the VSSSC is clearly superior to the other variable step-size algorithm (i.e., VSS).

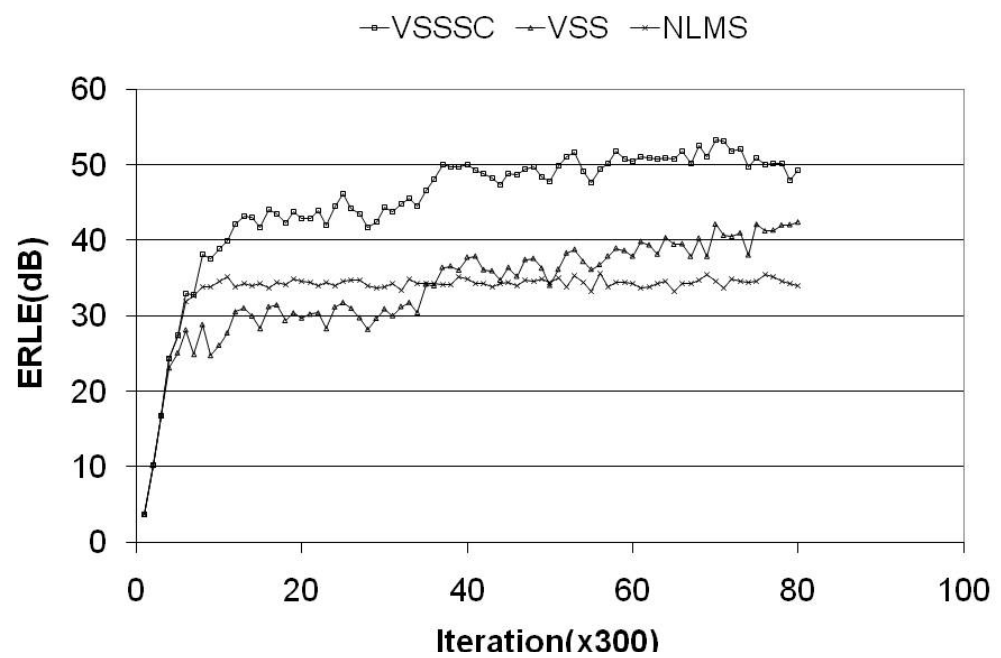

Figure 7. Comparison among the conventional NLMS, VSS, and VSSSC algorithms. The input signal was a voice signal. 


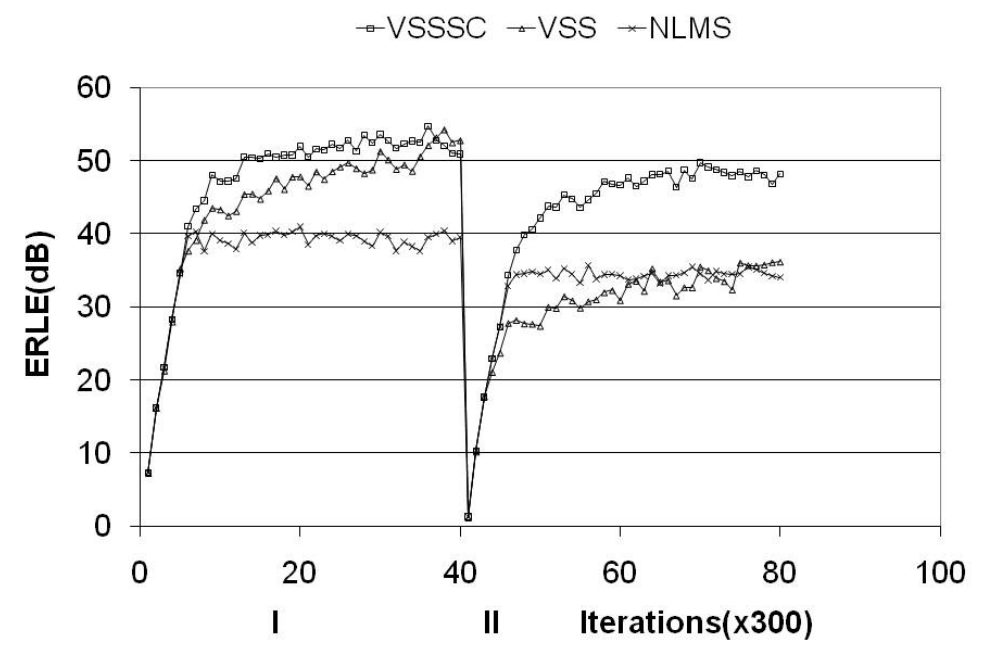

Figure 8. Comparison among the conventional NLMS, VSS, and VSSSC algorithms in two periods (I and II en the Figure). The input signal and additive noise were voice and white noise, respectively.

\section{Conclusions}

In this paper we introduced VSSSC, a variable step-size NLMS algorithm. The VSSSC algorithm updates the filter coefficients by taking into consideration the squared cross-correlation between the square of the output error $e(n)$ and the adaptive filter output $\hat{y}(n)$.

We carried out a performance comparison of the proposed algorithm against the conventional NLMS and VSS algorithms. In this paper, we provide supporting evidence regarding some advantages that we could identify in the proposed VSSSC algorithm. It achieves better ERLE than NLMS and VSS, and better convergence characteristics than VSS.

The proposed VSSSC algorithm can be used for updating the coefficients of a FIR structure in noise and echo canceling applications. 


\section{A New Variable Step-Size NLMS Algorithm and its Performance Evaluation in Echo Cancelling Applications, F. M. Casco-Sánchez et al. / 302-313}

\section{References}

[1] Widrow, B., McCool, J. M., Larimore, M. G. and Johnson, Jr., C.R., Stationary and Nonstationary Learning Characteristics of the LMS Adaptive Filter, Proceedings of the IEEE, vol. 64, no. 8, August 1976, pp 1151-1162.

[2] Haykin, S., Adaptive Filter Theory, 4th Ed., Prentice Hall, 2001.

[3] Raymond, H. K. and Johnston, E. W., A Variable Step Size LMS Algorithm, IEEE Transactions on signal processing, vol. 40, no. 7, July 1992, pp. 1633-1642.

[4] Mayyas, K. and Aboulnasr, T., A Robust Variable Step Size LMS-Type Algorithm: Analysis and Simulations, 1995 International Conference on Acoustics, Speech, and Signal Processing (ICASSP-95), 1995, vol. 2, pp.1408-1411, Detroit, Michigan, U.S.A., May.

[5] Wee-Peng A. and Farhang-Boroujeny B., A New Class of Gradient Adaptive Step-Size LMS Algorithms, IEEE Transactions of Signal Processing, vol. 49, no. 4, April 2001, pp. 805-810.

[6] Koike, S., A Class of Adaptive Step-Size Control Algorithms for Adaptive Filters, IEEE Transactions on Signal Processing, vol. 50, no. 6, June 2002, pp. 13151326.

[7] Yan L. and Xinan W., A Modified VS LMS Algorithm, Proceedings of the 9th International Conference on Advanced Communication Technology ICACT2007, 2007, pp. 615-618, Gangwon-Do, Korea (South), February.

[8] Itakura, H. and Nishikawa, Y., On Some Characteristics of an Echo Canceller using a learning identification algorithm, Trans. IEICE, vol. J60-A, num. 8, November 1977, pp. 1015-1022. 


\section{Authors' Biographies}

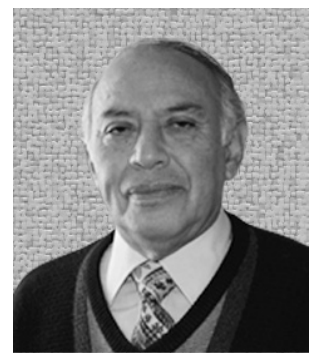

\section{Fausto M. CASCO-SÁNCHEZ}

Dr. Casco received the B.Sc. degree in electronics and communications in 1974 from Escuela Superior de Ingeniería Mecánica y Eléctrica (Higher Education School of Mechanical and Electrical Engineering), ESIMEZacatenco, and the M.Sc. degree in electrical engineering (with specialization in communications) in 1976 from Centro de Investigación y de Estudios Avanzados (Center for Research and Advanced Studies), CINVESTAV. In 1997, he received the doctoral degree from the Universidad Autónoma Metropolitana (Metropolitan Autonomous University), Iztapalapa Campus (UAM-I). From 1976-1977, he worked as a researcher for the Ministry of Communications and Transport. Since April 1977, he has been with the Departamento de Ingeniería Eléctrica (Department of Electrical Engineering) at the UAM-I, where he is a full time research professor. His research interests are digital communication systems, digital filter design, and adaptive filtering algorithms with applications to communication systems.

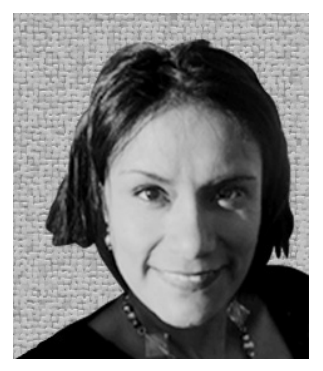

\section{R. Carolina MEDINA-RAMÍREZ}

She received her $\mathrm{PhD}$ degree in computer science from the Université de Niza Sophia-Antipolis and did her doctoral research at INRIA Sophia-Antipolis (The French National Institute for Research in Computer Science and Control), both in France. She is a researcher lecturer with Departamento de Ingeniería Eléctrica at Universidad Autónoma Metropolitana, Iztapalapa Campus. She has participated in the organization of several international congresses on artificial intelligence and computer science. Her research interests are corporate semantic WEB, information retrieval and P2P systems.

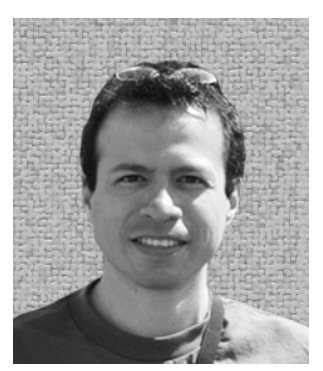

\section{Miguel LÓPEZ-GUERRERO}

He received his B.Sc. in mechanical-electrical engineering in 1995 and the M.Sc. in electrical engineering in 1998, both from Universidad Nacional Autónoma de México (National Autonomous University of Mexico, UNAM. He received his Ph.D. in electrical engineering from the University of Ottawa in 2004. He holds a position as a research professor with Universidad Autónoma Metropolitana, Iztapalapa Campus. His research interests are related to several aspects of communication networks such as traffic modeling, medium access control and performance evaluation. He has published over 30 research papers and regularly serves as member of the technical program committee for various international conferences. Dr. López is a SNI member and holds the PROMEP distinction. 